\title{
Sound Absorption of Palm Coir Fiber
}

\author{
Rahmad R. , Ahmad Sukri A.S. \\ * Faculty of Engineering Technology, Universiti Tun Hussien Onn Malaysia, Batu Pahat, Johor, Malaysia
}

Received 15 September 2018; accepted 1 December 2018; available online 30 December 2018

DOI: https://10.30880/jst.2018.10.04.010

\begin{abstract}
This research was conducted to study acoustic characteristic of palm coir fibre, which has high potential to be use for sound absorbing material. The sample was tested with different weight ratio. The weight ratio of palm coir fibre varied from $30 \mathrm{gm}$ to $60 \mathrm{gm}$ with $20 \mathrm{~mm}$ thickness. The samples were deformed by using the mould that was invented by using PVC pipe. These sample were tested by using Impedance tube according to international standard, ASTM E1050-98 used for measuring the sound coefficients. While for density and porosity test, the sample was tested according to international standard ASTM D792. The results showed sound absorption coefficients of palm coir fibre were good from $350 \mathrm{~Hz}$ to $2000 \mathrm{~Hz}$ within the range $0.65 \mathrm{~dB}-0.90 \mathrm{~dB}$ for Impedance tube. Results obtained for density and porosity test, also were good from $30 \mathrm{gm}$ and $60 \mathrm{gm}$ respectively. Overall, the optimum, sound absorption coefficient was obtained from weight $40 \mathrm{gm}$. These results indicate that palm coir fibre is promising to use as raw material of sound absorbing material with low cost, light and biodegradable.
\end{abstract}

Keyword: Sound Absorption; Palm Coir Fibre; Raw Material.

\subsection{Introduction}

Due to noise pollution in our surrounding, there are need and demand to find alternative materials that capable to reduce the noise level at various frequency ranges. Research on composite materials and natural fibres were done on acoustical panels. The common acoustical panels made from synthetic fibres that are hazardous to human health and environment and quite expensive for small portion. Therefore, some researchers showed their great interest to make alternative sound absorber from recycled materials, such as textile, foam, rubber or plastic.

A study on acoustical characteristics and physical-mechanical properties of plaster with rubber waste additives were also done [1]. The sound absorbent material made from scrap tires with small grains yields showed better sound absorption [2]. Even product made from recycled are welcomed, issues like energy consumption during producing and environment impact of binders need were, considered. Thus, alternative raw materials those are of low cost, renewable, plentiful, and save for environment and human health is needed.

Moreover, many studies focused in developing natural fibres as raw material were done and reported. Paddy straw, kenaf, coconut coir, and many others that have potential used as raw material of acoustical panel. Paddy straw was reported suitable for acoustic panel because of its high elasticity and hollow space [3, 4]. A single layer acoustic panel made of paddy husk reinforced sodium silicate showed the optimum, sound absorption coefficient at higher silicate content in high range frequencies [5]. Similarly, coconut and jute fibre have high potential used as a sound absorber material [6]. Coconut coir fibre has good sound absorption at higher frequencies but less for the lower frequencies. These show that natural fibres have high potential to be applied as raw material of sound absorbing materials. However, sound absorption characteristic of some natural fibre was investigated and the acoustical characteristics of palm frond coir fibre, is rarely studied.

Palm frond coir fibre is one of natural fibres that abundantly available from palm tree. Since the last decade, it is widely used for many applications like roof, rope, water filter, and sound proof in recording studio [7]. In this research, the fibres were obtained from palm frond. The fibres were treated by using Sodium Chloride $(\mathrm{NaOH})$ substrate for 24 hours as their pre-treatment. This treatment mercerized the fibres, removed the lignin and strengthened the fibres. Then, the fibres were mixed with epoxy in the custom PVC pipe mould to produce 
samples. Next, these samples were tested by using Impedance tube test (ASTM E1050-98) for measuring the sound coefficients. Thus for density and porosity test, the samples were tested by using density test (ASTM D792). Based on the tests above, the samples were analysed.

\subsection{Methodology}

This section discussed on the methodology used in this study that propose using palm coir fibre as sound absorption. Fig. 1 shows the flow chart of methodology. It starts with fibre, mould and samples preparation. Next, sound absorption test and data collection were performed.

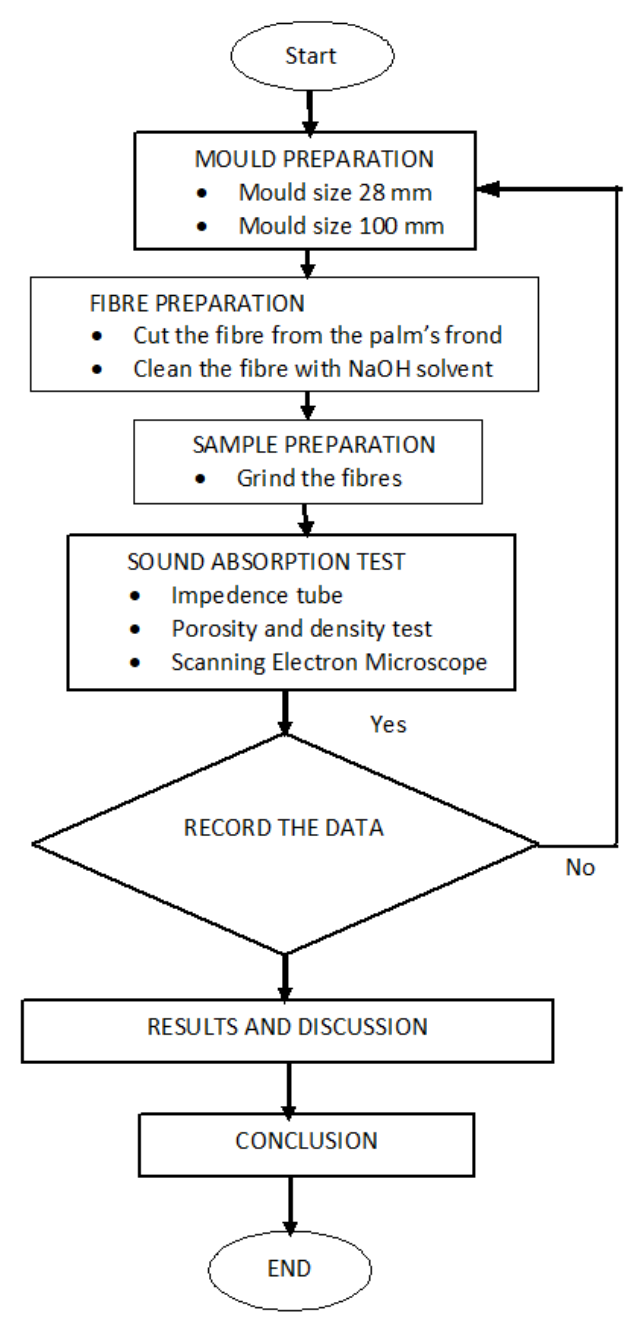

Fig. 1 Flow chart of the methodology
The material used in this study is palm coir fibre made from palm tree frond. The fibres were cleaned with Sodium Hydroxide $(\mathrm{NaOH})$ and water then dried in room temperature for 24 hours. Next, the fibres were grinded into a small piece. Then, the dried fibres were coated with Epoxy Resin and deformed using custom PVC mould sized $100 \mathrm{~mm}$ and $28 \mathrm{~mm}$. After the samples dried, the fibre was cut down in cylindrical shape according to size of the Impedance tube. Two different diameter tubes were used to cover the full frequency range. Tube with $100 \mathrm{~mm}$ diameter was used to cover measurements over the frequency range from $350 \mathrm{~Hz}$ to $2000 \mathrm{~Hz}$ and $28 \mathrm{~mm}$ diameter tube was used to cover measurements in the frequency range $750 \mathrm{~Hz}$ to $6400 \mathrm{~Hz}$.

The acoustic property of palm coir fibre was studied in this work is sound absorption coefficient $(\alpha)$. Impedance Tube Method (ASTM E1050-98) was used to measure the sound absorption coefficient. This method began with placed a loudspeaker at one end of an impedance tube and a small cylindrical sample of the material at the other end. The loudspeaker generated a broadband, stationary random sound based on the coefficient of these four samples. The sound waves propagated within the tube, strike the sample and reflected, resulting in a standing wave interference pattern. The sound absorption measurement was done by varied the volume of palm coir fibre $30 \mathrm{gm}, 40 \mathrm{gm}, 50 \mathrm{gm}$ and $60 \mathrm{gm}$ in $2 \mathrm{~cm}$ of thickness. The measurement process was done from frequency $350 \mathrm{~Hz}-6400 \mathrm{~Hz}$.

For density measurement, density test machine (ASTM D792) was used. Every fibrous material containing their own density. High-density material related with fibre size. The size of palm fibres influenced the absorption sound coefficient. Smaller the size of fibre will increase coefficient of sound absorption [8]. Another measurement using the same machine is to measure the porosity test. Most of the porous sound-absorbing materials commercially available are fibrous. Fibrous materials are composed of a set of continuous filaments that trap air between them [9]. The porosity test determined the volume of air trapped in the void of the samples. The air in the sample affected the coefficient of sound absorption due of empty volume in the samples. In this project samples were tested in four different types of weight. The porosity determined by using the formula in Eq. 1 . 


$$
\text { Porosity }=\frac{\left(\mathrm{w}_{\mathrm{w}}-\mathrm{w}_{\mathrm{s}}\right)-\left(\mathrm{w}_{\mathrm{d}}-\mathrm{w}_{\mathrm{s}}\right)}{\mathrm{w}_{\mathrm{w}}-\mathrm{w}_{\mathrm{S}}}
$$

Where,

$\mathrm{W}_{\mathrm{w}}=$ weight of samples

$\mathrm{w}_{\mathrm{d}}=$ weight of disposal samples

$\mathrm{w}_{\mathrm{s}}=$ weight of dry samples

For examine the structure of the samples palm coir fibre, the scanning electron microscope (SEM) was used to scan any contamination in the samples. SEM is one of the most versatile instruments available for the examination and analysis of the microstructure morphology and chemical composition characterizations. The term contamination describes the collective phenomena by which the surface of samples undergoes deposition of a foreign substance, generally a carbonaceous material derived from the breakdown of hydrocarbon. For this test, the samples need to cut into dice size. The size shown in Fig. 2.

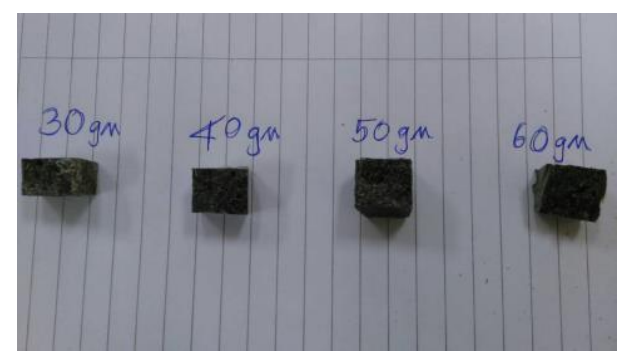

Fig. 2 Dice size samples for SEM test

\subsection{Results and Discussion}

The sound absorption coefficient of palm coir fibre measured from the impedance tube ranges from low to high frequencies are as shown in Fig. 3. In this study, the volume of fibres were, varied that are $30 \mathrm{gm}, 40 \mathrm{gm}, 50 \mathrm{gm}$ and $60 \mathrm{gm}$. It shows that the palm coir fibre is a good absorber at optimum, sound absorption coefficient $0.90 \mathrm{~dB}$ for $40 \mathrm{gm}$ weight of fibre. Physically good absorber is a common property of fibrous material. The weight of fibre had influence to sound absorption coefficient of the material. These data show otherwise, the maximum weight material exhibits a maximum sound absorption coefficient at high frequencies but in this data, shows the less weight absorb perfectly than the maximum weight.

\section{SOUND ABSORPTION COEFFICIENT}

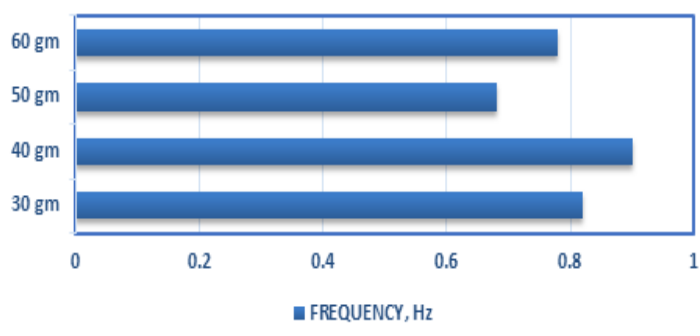

Fig. 3 Results sound absorption

According to theory, epoxy are better than the other adhesives due its attribute to absorb the deflected sound. Furthermore, epoxy eliminate the vibration and sound until reaches $0.7 \mathrm{~dB}$ which makes this sample better than the other samples that contain low volume of epoxy resin.

Porosity and density data were collected Fig. 4 and Fig. 5 show average data for porosity and density value for these samples at four different weight.

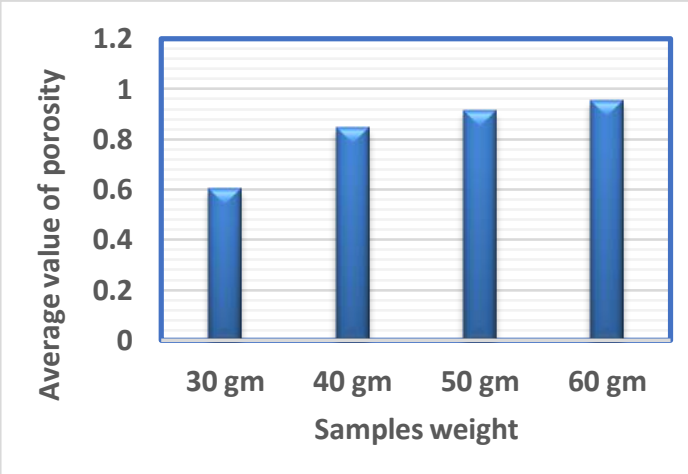

Fig. 4 Results for porosity value

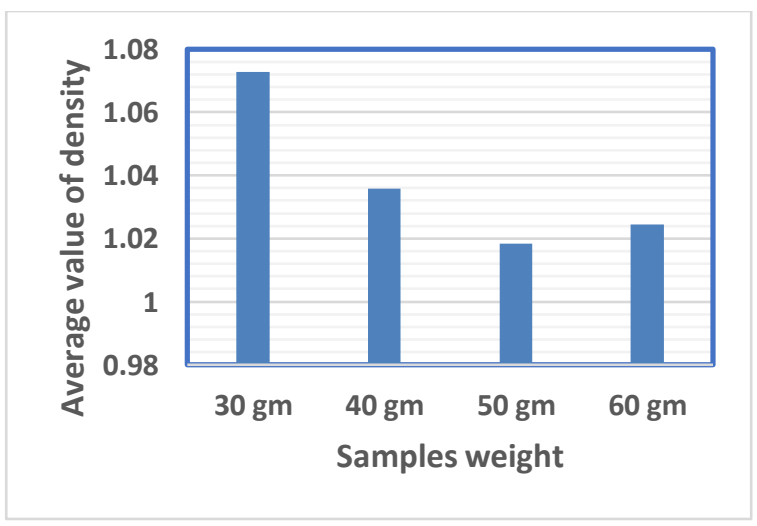

Fig. 5 Results for density value 
The average porosity for four different samples using palm coir fibre coated with epoxy resin, shown in Fig. 4 indicates a good porosity in sample of weight $60 \mathrm{gm}$ having a value $0.953 \mathrm{PU}$, a better porous material than the others do. This related with the volume of fibre in the samples. The volume of fibre affected the samples and it became most porous material for the samples. Good porous material leads to the great sound absorption that the pores in fibrous material make the loss of sound waves due to the increase of surface friction, which leads to an increase in sound absorption performance. In this research, epoxy resin absorbs deflected sound higher than the porous material.

Fig. 5 shows the average density for four different samples. The density of a material is often a significant factor governing its sound absorption qualities. Density related with the size of the fibres. The amount of fibre increases in the number per unit area, the sample density increases [10]. Sample for $30 \mathrm{gm}$ which value $1.0729 \mathrm{~g} / \mathrm{cm}^{3}$ shows it have the maximum density rather than the other due of the least numbers of fibres contain on the sample.

SEM test was conducted to determine the material structure and contamination in the samples. Fig. 6a, 6b, 6c, and $6 \mathrm{~d}$ show the structure for four different palm coir fibre, samples.

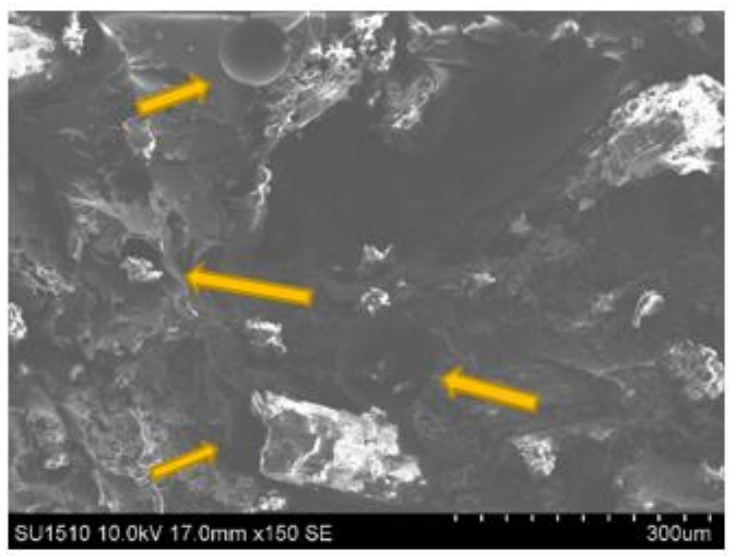

Fig. 6a Structure for samples $30 \mathrm{gm}$

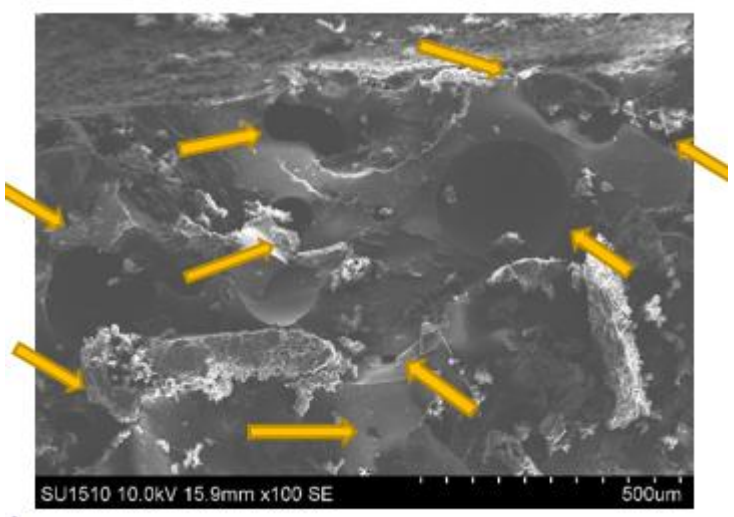

Fig. 6b Structure for samples $40 \mathrm{gm}$

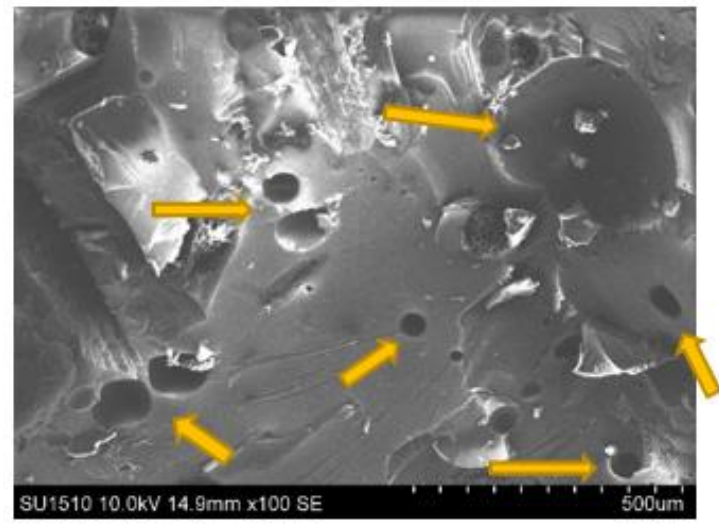

Fig. 6c Structure for samples $50 \mathrm{gm}$

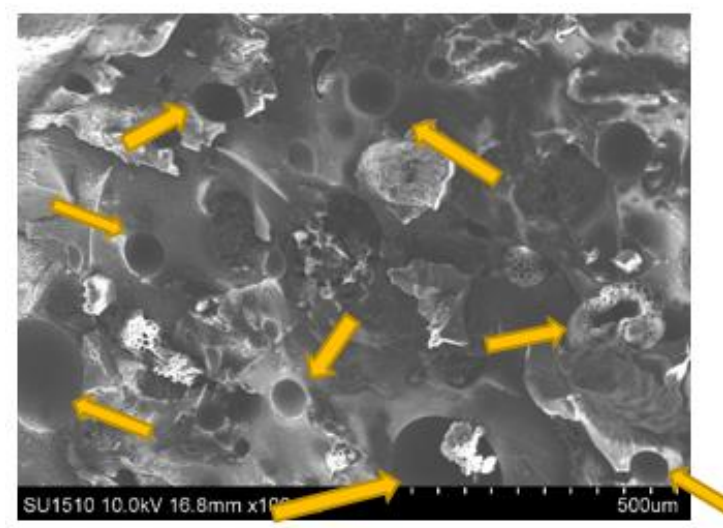

Fig. 6d Structure for samples $60 \mathrm{gm}$

The voids in the figures (yellow arrow) prove the samples is a porous material. The porous material effectively helps in sound absorption compare to flat material. More porous in the sample affect the sound absorption coefficient. Based on the figures, the samples in $40 \mathrm{gm}$ have more voids in the sample which it affects the rate of efficiency of sound absorption. Moreover, the scanning electron microscope functioned to detect any contamination in the structure like insects. 


\subsection{Conclusion}

This research investigated the potential of using palm coir fibre as raw material for sound absorbing material. Palm coir fibre coated with epoxy resin was tested by using Impedance tube according to international standard that is ASTM E1050-98 purposely for measuring the sound coefficients. For density and porosity test sample was tested using density test according to international standard ASTM D792. The optimum, sound absorption coefficient of palm coir fibre was obtained from $40 \mathrm{gm}$ weight. The sound absorption coefficients were good from the medium to low frequency that is from 630 $\mathrm{Hz}$ to $2000 \mathrm{~Hz}$ within the range of $0.65 \mathrm{~dB}-$ $0.90 \mathrm{~dB}$. These results indicate that palm coir fibre is promising to be used as raw material of sound absorbing material that low cost, less weight, and biodegradable.

\section{Acknowledgements}

We would like to thanks University Tun Hussein Onn Malaysia for sponsor and financial support in publishing this paper.

\section{References}

[1] Vytautas S., Gintautas S., Audrius G. and Kestutis M., (2007) Acoustical characteristics and physical - Mechanical properties of Plaster with Rubber Waste Additives, Material Science, Vol. 13 No. 4, pp. 304-309.

[2] Poulain, N. et. al. (2006) Development of an Acoustic Absorbent Material Using Scrap Tires, CTTM-IRC, 2006.

[3] Christina, E. M. (2007) Potential of Paddy Straw as Material Raw of Acoustic Panel, Architecture Dimension, Vol. 35 No.2, pp. 183-189.

[4] Christina, E. M., (2008) Paddy Straw as Walling Panel, Architecture Dimension, Vol 36 No. 1, pp. 20-27.

[5] Ridha, F., (2006) Develop single layer acoustic sample made of paddy husk and normal absorption characteristics, Institut Teknologi Bandung: Undergraduate Theses
[6] Sabri, (2007) Evaluation of the Acoustical Performance of Natural Fiber as Alternative Material to Control Noise, Institut Teknologi Bandung: Master Theses.

[7] Lindawati, I. et.al. (2009) Medium Density fiberboard: Potential Application as an Acoustical Panel, Proceeding of Icon BSE 09-67, Malaysia.

[8] Hasina, M.et.al, (2016) Acoustic Absorption of Natural Fiber Composites, Journal of Engineering, Vol. 7, No.1, pp. 111.

[9] Attenborough, K. (1993) Models for the acoustical characteristics of ai filled granular materials, Acta Acustica, Vol. 1 No.4, pp. 213-226.

[10] Koizumi, T. et.al., (2002) The development of sound absorbing materials using natural bamboo fibers high Performance, WIT Press, United Kingdom, Vol. 59, No. 1, pp. 1-10. 